\title{
Line blot immunoassays in idiopathic inflammatory myopathies: retrospective review of diagnostic accuracy and factors predicting true positive results
}

Fergus To ${ }^{1}$ (D), Clara Ventín-Rodríguez ${ }^{2}$, Shuayb Elkhalifa ${ }^{3}$, James B. Lilleker ${ }^{4,5}$ (D) and Hector Chinoy ${ }^{6,7,8^{*}}$ (D)

\begin{abstract}
Background: Line blot immunoassays (LIA) for myositis-specific (MSA) and myositis-associated (MAA) autoantibodies have become commercially available. In the largest study of this kind, we evaluated the clinical performance of a widely used LIA for MSAs and MAAs.
\end{abstract}

Methods: Adults tested for MSA/MAA by LIA at a tertiary myositis centre (January 2016-July 2018) were identified. According to expert-defined diagnoses, true and false positive rates were calculated for strongly and weakly positive autoantibody results within three cohorts: idiopathic inflammatory myopathy (IIM), connective tissue disease (CTD) without myositis, and non-CTD/IIM. Factors associated with true positivity were determined.

Results: We analysed 342 cases. 67 (19.6\%) had IIM, in whom 71 autoantibodies were detected (50 strong positives [70.4\%], 21 weak positives [29.6\%]). Of the strong positives, 48/50 (96.0\%; 19 MSAs, 29 MAAs) were deemed true positives. Of the weak positives, 15/21 (71.4\%; 3 MSAs, 12 MAAs) were deemed true positives.

In CTD without myositis cases ( $n=120), 31 / 61$ (51.0\%; 5 MSAs, 26 MAAs) autoantibodies were strongly positive, with 24/31 (77.4\%; 0 MSAs, 24 MAAs) true positives. 30/61 (49.2\%; 13 MSAs, 17 MAAs) were weakly positive, with 16/30 (53.3\%; 0 MSAs, 16 MAAs) true positives. In non-CTD/IIM cases $(n=155)$, all 24 MSAs and 22 MAAs were false positives; these results included 17 (37.0\%; 7 MSAs, 10 MAAs) strong positives.

Individual autoantibody specificities were $>98.2$ and $>97.5 \%$ for weakly and strongly positive results, respectively. True positivity was associated with high pre-test for IIM (odds ratio 50.8, 95\% Cl 13.7-189.2, $p<0.001$ ) and strong positive (versus weak positive) results $(4.4,2.3-8.3, p<0.001)$.

Conclusions: We demonstrated the high specificity of a myositis LIA in a clinical setting. However, a significant burden of false positive results was evident in those with a low pre-test likelihood of IIM and for weakly positive autoantibodies.

Keywords: Immunoblotting; myositis; autoantibodies, Inflammatory muscle disease, Idiopathic inflammatory myopathy, Line blot immunoassay

\footnotetext{
* Correspondence: hector.chinoy@manchester.ac.uk

${ }^{6}$ National Institute for Health Research Manchester Biomedical Research Centre, Manchester University NHS Foundation Trust, The University of Manchester, Manchester, UK

${ }^{7}$ Department of Rheumatology, Salford Royal NHS Foundation Trust, Manchester Academic Health Science Centre, Stott Lane, Salford M68HD, UK Full list of author information is available at the end of the article
}

C C The Author(s). 2020 Open Access This article is licensed under a Creative Commons Attribution 4.0 International License, which permits use, sharing, adaptation, distribution and reproduction in any medium or format, as long as you give appropriate credit to the original author(s) and the source, provide a link to the Creative Commons licence, and indicate if changes were made. The images or other third party material in this article are included in the article's Creative Commons licence, unless indicated otherwise in a credit line to the material. If material is not included in the article's Creative Commons licence and your intended use is not permitted by statutory regulation or exceeds the permitted use, you will need to obtain permission directly from the copyright holder. To view a copy of this licence, visit http://creativecommons.org/licenses/by/4.0/. The Creative Commons Public Domain Dedication waiver (http://creativecommons.org/publicdomain/zero/1.0/) applies to the data made available in this article, unless otherwise stated in a credit line to the data. 


\section{Background}

The idiopathic inflammatory myopathies (IIM) are heterogeneous multisystem autoimmune diseases often presenting with skeletal muscle weakness, rash, arthritis, and/or interstitial lung disease [1]. IIM subtypes include immune-mediated necrotising myopathy (IMNM), sporadic inclusion-body myositis (IBM), overlap myositis (OM), anti-synthetase syndrome (ASS), dermatomyositis (DM) and polymyositis (PM) [1-5].

Approximately $2 / 3$ of IIM patients have detectable serum autoantibodies (Abs), termed myositis-specific autoantibodies (MSA), which are unique to IIM and usually mutually exclusive to one another, or myositisassociated autoantibodies (MAA) which can occur in other connective tissue diseases (CTD) [6-8]. MSA/ MAAs are gaining importance in IIM diagnosis, can circumvent the requirement for a muscle biopsy, and may inform prognosis [9-18].

Line blot immunoassays (LIA) for MSA/MAA have become commercially available, increasing the availability of testing in clinical practice [19]. Literature suggests LIA is an appropriate substitute to conventional immunoprecipitation for MSA/MAA testing, but only small samples have been studied with variable accuracy demonstrated $[13,19,20]$.

In the largest study of its kind, we evaluated the diagnostic accuracy of a commercially available LIA for MSA and MAA testing in a clinical setting and examined factors associated with true positive results.

\section{Methods}

\section{Cases}

We retrospectively identified patients tested with the EUROLINE Inflammatory Myopathies 16 Ag (IgG) commercial LIA (Euroimmun, Lubeck, Germany) from January 1st, 2016, to July 30th, 2018, at Salford Royal NHS Foundation Trust (SRFT), United Kingdom. The search was limited to patients being reviewed in tertiary IIM, systemic sclerosis and neuromuscular outpatient clinics. Patients without available clinical data were removed. Where duplicate testing occurred, only the most recent results were analysed.

This study was performed as part of a quality improvement project evaluating LIA usage at SRFT. Case notes and other data were reviewed retrospectively without alteration to patient management. Given this context, and after consultation with the Health Research Authority (via www.hra-decisiontools.org.uk) this study proceeded without further requirement for ethical authorization.

\section{Autoantibody testing}

The studied LIA can detect 12 MSAs (anti-Mi2A, antiMi2B, anti-TIF1 $\gamma$, anti-MDA5, anti-NXP2, anti-SAE1, anti-SRP, anti-Jo1, anti-PL7, anti-PL12, anti-EJ, anti-OJ) and 4 MAAs (anti-Ku, anti-PM-Scl100, anti-PM-Scl75, anti-Ro52). Laboratory testing was performed using the scanner, software, and protocol provided by the manufacturer. Results were categorised as negative, weakly positive, or strongly positive according to the signal intensity measured digitally as per the manufacturer's specifications.

\section{Clinical data collection methodology}

Patient records were reviewed by 2 authors (FT and CR) independently. Demographics, electromyography (EMG) results, muscle biopsy results, and peak serum total creatine kinase (CK) levels were collated. Indications for ordering the LIA (pre-test diagnoses) were categorised retrospectively as "suspected IIM", "CTD without evidence of myositis", or "myopathic syndromes with low likelihood of IIM". The final diagnosis made by the expert treating clinician was recorded and categorised retrospectively as "IIM" (including overlap syndromes), "CTD without myositis", or "non-IIM/CTD". Categorisation was agreed on between the authors (FT, CR), and a third author was consulted in indeterminate cases (JBL or $\mathrm{HC}$ ). Diagnoses were verified through review of extensive clinical information reflecting several years of care in each case. Research classification criteria were not applied as by their nature these are restrictive and would limit the real-world applicability of this study. In addition, recent studies have demonstrated classification criteria may not accurately reflect clinical diagnoses made by expert clinicians [21].

\section{Assay performance}

$\mathrm{Ab}$ results were reviewed and categorised as true or false positive according to the available clinical information. True positive MSAs were defined as those in patients diagnosed with IIM with the phenotype and IIM subtype expected of that MSA. True positive MAAs were defined as those in patients diagnosed with either CTD or IIM phenotypes expected of that MAA. Otherwise, results were deemed to be false positives. For patients with multiple MSAs, that which best reflected the clinical phenotype was assigned true positive, as MSAs are generally mutually exclusive [8]. Additional MSAs in such cases were false positives, except for anti-Mi2A and anti-Mi2B (isoforms of the same $\mathrm{Ab}$ ) where simultaneous true positives were accepted [22, 23]. All negative results were deemed true negatives. In cases of uncertainty, an immunologist (SE) reviewed the source data to ensure accuracy.

\section{Statistics}

Analysis was performed with STATA version 14 (College Station, USA). Descriptive statistics examined characteristics of different groups according to $\mathrm{Ab}$ status. 
Categorical data were summarised as frequencies and proportions. Continuous data were summarised using means and standard deviations. For individual Abs, the rate of true and false positivity and the associated specificity for the presence of a consistent diagnosis or disease subtype was calculated. Logistic regression was performed to investigate factors associated with true positive results. A $p$-value $<0.05$ represented a statistically significant difference.

\section{Results}

\section{Patient characteristics}

In total, 401 LIAs were performed in 394 patients. Clinical data were missing for 55 cases and duplicate testing occurred in 7 patients (Fig. 1). Of the remaining 342, IIM was diagnosed in $67 / 342(19.6 \%)$ patients, CTD without myositis in $120 / 342(35.1 \%)$ patients, and nonCTD/IIM in 155/342 (45.3\%) patients (Table 1).

\section{Autoantibody profiles in IIM patients: Most weak positive MSAs were false positives}

At least $1 \mathrm{Ab}$ was detected in 42/67 (62.7\%) IIM patients (Table 2). Their clinical phenotypes included DM $(n=$ $11)$, IMNM $(n=11)$, ASS $(n=7)$, clinically amyopathic dermatomyositis $(n=4)$, PM $(n=4)$, and IBM $(n=2)$. In these 42 patients, a total of 71 Abs were detected (21 patients had multiple Abs; Fig. 2). 50/71 (70.4\%) Abs were strongly positive (21 MSAs, 29 MAAs), and the majority of these were true positives (48/50 [96.0\%]). 2/50 strongly positive Abs were false, and both were MSAs (anti-PL7 and anti-SAE1). Both patients had ASS and each had another concurrently detected MSA that was deemed to be the true positive. There were 21/71 (29.6\%) weak positive Abs (7 MSAs, 14 MAAs). Of these, $15 / 21(71.4 \%)$ were true positives (3 MSAs, 12 MAAs) and 6/21 (28.6\%) were false positives (4 MSA, 2 MAAs).

Seven IIM patients had apparent dual MSA positivity. 3/7 (43.0\%; DM $(n=2)$ and OM $(n=1))$ had concurrent anti-Mi2A and anti-Mi2B. 1/7 $(14.3 \%$; ASS $(n=1))$ was strongly positive for both anti-EJ and anti-PL7; anti-EJ was deemed the true positive based on significantly higher signal intensity when reviewed with the source data compared to anti-PL7 which just met the cut off for a strong positive result. Another was strongly positive for both anti-Jo1 and anti-SAE1; anti-Jo1 was felt to be the true positive as the patient clinically had ASS. 2/7 (28.6\%) patients had dual weak false positives (antiMDA5 and anti-TIF1 $\gamma$ in a patient with OM; anti-SAE1 and anti-SRP in a patient with PM).

\section{Autoantibody profiles in CTD patients without myositis: False positive MSAs are detected commonly}

At least $1 \mathrm{Ab}$ was detected in 41/120 (34.2\%) patients with CTD without myositis (Fig. 2 and Table 2). Systemic sclerosis was the most common diagnosis. A total

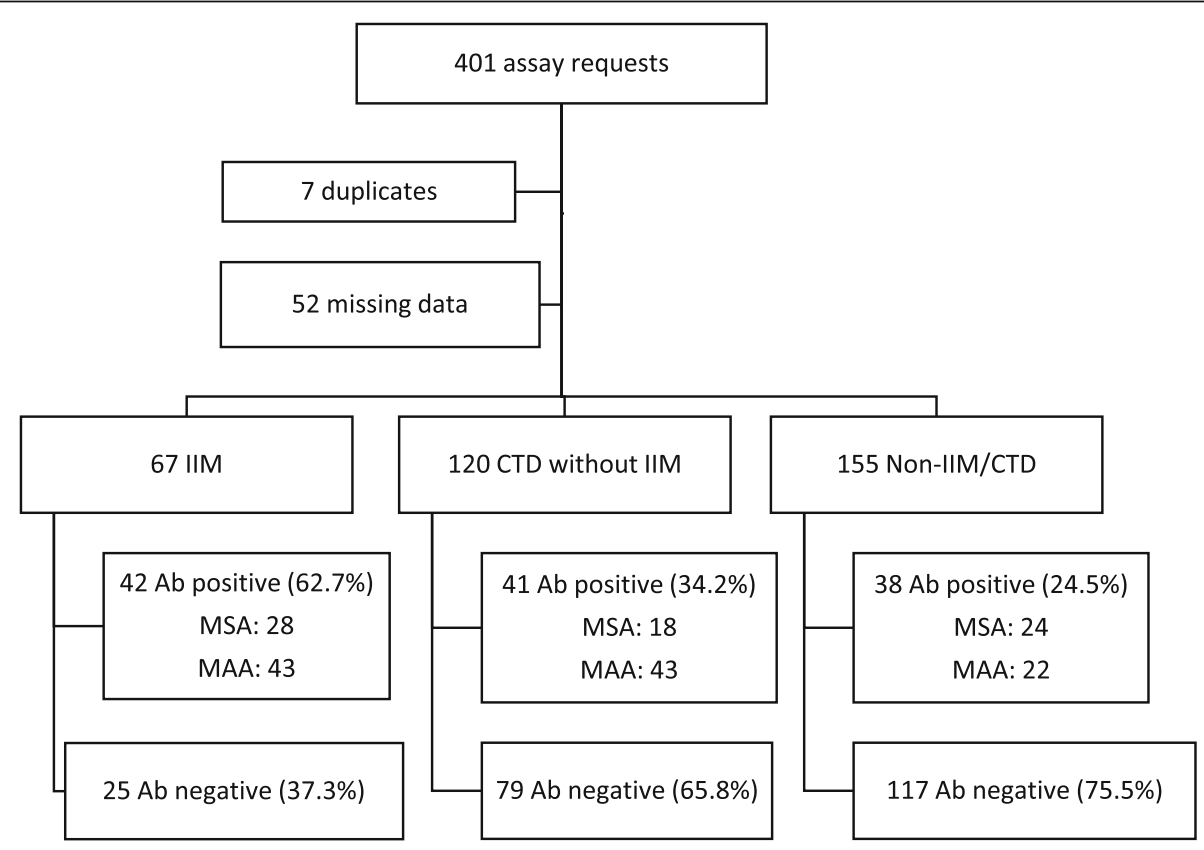

IIM: idiopathic immune-mediated myopathy; CTD: connective tissue disease; MSAs: myositis-specific autoantibodies; MAAs: myositis-associated autoantibodies; Ab: autoantibodies.

Fig. 1 Study population categorised by final diagnoses and total number of Abs detected in each group 
Table 1 Characteristics of study population

\begin{tabular}{|c|c|}
\hline \multicolumn{2}{|l|}{ Number of patients } \\
\hline Total & 342 \\
\hline Mean age in years (SD) & $54(14)$ \\
\hline Female gender & $222(65 \%)$ \\
\hline \multicolumn{2}{|l|}{ Pre-test working diagnosis } \\
\hline Suspected IIM & $92(26.9 \%)$ \\
\hline CTDs without IIM & $137(40.1 \%)$ \\
\hline Myopathic syndromes with low likelihood of IIM & $113(33.0 \%)$ \\
\hline \multicolumn{2}{|l|}{ Final diagnosis and subtype } \\
\hline$\| \mathrm{M}$ & $67(19.6 \%)$ \\
\hline Overlap myositis & 21 \\
\hline Dermatomyositis & 12 \\
\hline Polymyositis & 11 \\
\hline Antisynthetase syndrome & 7 \\
\hline Amyopathic dermatomyositis & 7 \\
\hline Inclusion body myositis & 5 \\
\hline Immune mediated necrotizing myopathy & 4 \\
\hline CTD without IIM & $120(35.1 \%)$ \\
\hline Systemic sclerosis & 91 \\
\hline Undifferentiated CTD & 10 \\
\hline Systemic lupus erythematosus & 7 \\
\hline Inflammatory arthritis & 6 \\
\hline Overlap CTD & 4 \\
\hline Sjogrens syndrome & 2 \\
\hline Non-IIM/CTD & $155(45.3 \%)$ \\
\hline Other rheumatologic diagnoses & 45 \\
\hline Other neurologic diagnoses & 12 \\
\hline Genetic myopathy & 11 \\
\hline Endocrinologic myopathy & 5 \\
\hline Idiopathic pulmonary fibrosis & 3 \\
\hline Post-viral myopathy & 3 \\
\hline Traumatic myopathy & 3 \\
\hline Familial amyloidosis & 1 \\
\hline Orbital myositis & 1 \\
\hline Malignancy & 1 \\
\hline Unclear & 70 \\
\hline
\end{tabular}

SD standard deviation, IIM idiopathic inflammatory myopathy, CTD connective tissue disease

of 61 Abs were detected these 41 individuals with 31/61 (50.8\%) being strongly positive; $24 / 31$ (77.4\%) of these were true strong positives, and all were MAAs (anti-Ro52 $[n=20]$, anti-PMScl100 [ $n=1]$, anti-PMScl75 $[n=1]$, anti-Ku $[n=2])$. Of the strong false positive Abs $(n=7), 5$ were MSAs ( $n=1$ for each of anti-EJ, anti-Mi2A, antiSAE1, anti-SRP, anti-PL12), and 2 were MAAs (anti-Ku in a patient with resolved undifferentiated connective tissue
Table 2 Cases of antibody positivity by final diagnosis

\begin{tabular}{ll}
\hline IIM & $42 / 67(62.7 \%)$ \\
Only weak MSA/MAA & $9(13.4 \%)$ \\
Only strong MSA/MAA & $27(40.3 \%)$ \\
Both weak and strong MSA/MAA & $6(9.0 \%)$ \\
CTD without IIM & $41 / 120(34.2 \%)$ \\
Only weak MSA/MAA & $13(10.8 \%)$ \\
Only strong MSA/MAA & $18(15.0 \%)$ \\
Both weak and strong MSA/MAA & $10(8.3 \%)$ \\
Non-IIM/CTD & $38 / 155(24.5 \%)$ \\
Only weak MSA/MAA & $22(14.2 \%)$ \\
Only strong MSA/MAA & $13(8.4 \%)$ \\
Both weak and strong MSA/MAA & $3(1.9 \%)$ \\
Any positive MSA/MAA across all diagnostic groups & $121(35.4 \%)$ \\
\hline
\end{tabular}

IIM idiopathic inflammatory myopathy, CTD connective tissue disease, MSA myositis-specific autoantibody, MAA myositis-associated autoantibody

disease and anti-PM-SCL75 in a patient with systemic lupus erythematosus).

Weakly positive Abs were more common in CTD patients without myositis (30/61 [49.1\%]; 13 MSAs, 17 MAAs) compared to IIM patients (21/71 [29.6\%]; 7 MSAs, 14 MAAs; $p=0.02$ ). 16/30 (53.3\%) were true positives (all MAAs), and of the remaining false positives, 1 MAA and 13 MSA were detected (anti-SRP [ $n=4]$, antiPL12 $[n=2]$, anti-PL7 [ $n=2]$, anti-TIF1 $\gamma[n=2]$, anti-Jo $[n=1]$, anti-Mi2A $[n=1]$, and anti-OJ $[n=1])$.

Four patients in the CTD group without myositis had multiple MSAs. All were false positives, and all had an anti-SRP (anti-SRP with anti-OJ, anti-SRP with antiPL12, anti-SRP with anti-EJ, and anti-SRP with both anti-Mi2A anti-PL12).

Autoantibody profiles in non-IIM/CTD patients: Detection of MSAs and MAAs is common

At least $1 \mathrm{Ab}$ was detected in 38/155 (24.5\%) non-IIM/ CTD patients with a total of 46 detected Abs in these 38 individuals (24 MSAs [52.2\%], 22 MAAs [47.8\%]) (Fig. 2 and Table 2). All were deemed to be false positive results. 17/46 (37.0\%) Abs were strong positives and 7/17 (41.2\%) of these were MSAs. The final diagnoses for the 6 patients with strongly positive MSAs deemed to be false positives included immune checkpoint inhibitor related myofasciitis $(n=1$; anti-EJ), genetic myopathy $(n=$ 1 ; anti-Mi2B with anti-PL7), fibromyalgia ( $n=1$; antiJo1), and unclear final diagnoses $(n=3$; anti-Mi2B, antiTIF1 $\gamma$, and anti-EJ).

29/46 (63.0\%) Abs were weakly positive and 17/29 of these were MSAs. In these, the final diagnoses were immune checkpoint inhibitor related myofasciitis $(n=1$; anti-SRP), primary Raynaud's phenomenon $(n=1$; antiTIF1 $\gamma$ ), fibromyalgia ( $n=1$; anti-Mi2B), statin-related 


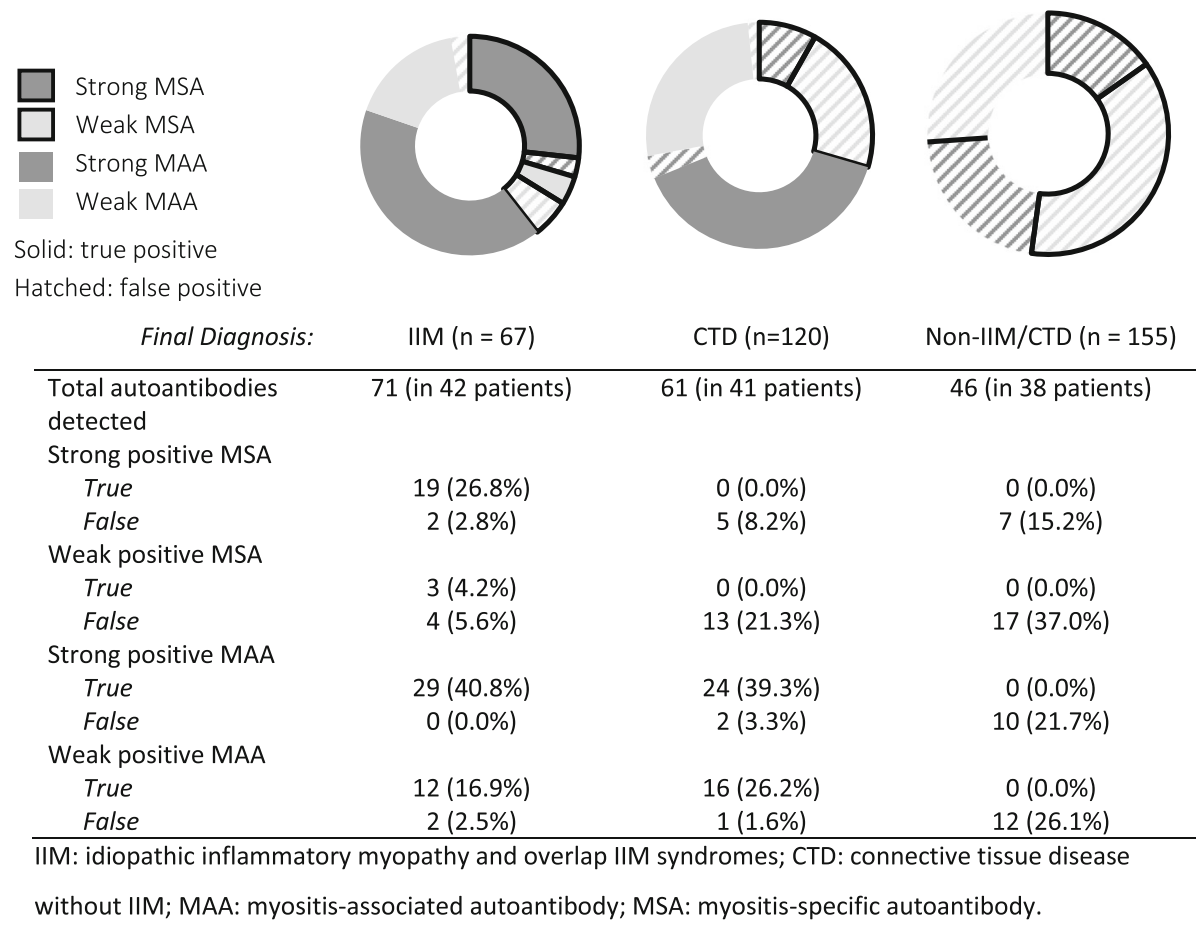

Fig. 2 Categorisation of autoantibodies by final diagnosis, subtype, and strength of result

toxic myopathy ( $n=1$; anti-Mi2B with anti-SAE1), polymyalgia rheumatica $(n=2$; anti-OJ, anti-PL7), familial amyloidosis $(n=1$; anti-SAE1), and unclear $(n=8$; antiSRP $[n=3]$, anti-TIF1Y $[n=2]$, antiPL7 with anti-SRP $[n=1]$, anti-Mi2A with anti-SRP $[n=1]$, anti-PL12 with anti-SRP $[n=1])$.

Apparent dual positive MSAs were seen in 4 cases: anti-SRP with anti-PL12, anti-SRP with anti-Mi2B, anti$\mathrm{Mi} 2 \mathrm{~B}$ with anti-SAE1, and anti-PL7 with anti-Mi2B.

\section{Factors associated with true and false positive antibody results \\ Weakly positive MSAs are often false positives}

Across all diagnostic groups, weak positive MSAs were more likely to be false positives than true positives (34/ 37 [91.9\%] false positives vs. 3/37 [8.1\%] true positives), although the same was not true for weak positive MAAs (15/43 [34.9\%] false positives vs. 28/43 [65.1\%] true positives). This contrasts with strongly positive results, where the majority were true positives (19/33 [57.6\%] true positives for MSAs, 53/65 [81.5\%] for MAAs).

All weak positive anti-SRP $(n=10)$, anti-TIF1 $\gamma(n=6)$, anti-SAE1 $(n=3)$, anti-PL7 $(n=3)$, anti-OJ $(n=2)$, antiMi2B $(n=2)$, anti-Jo1 $(n=1)$, and anti-MDA5 $(n=1)$ were false positives. For anti-PL12, false weak positivity was found in $4 / 5(80.0 \%)$ cases. Amongst MAAs, false weak positives were found in $3 / 4(75.0 \%)$ cases of anti$\mathrm{Ku}$ and 6/14 (42.9\%) cases of anti-Ro52. Anti-PM-Scl100 and anti-PM-Scl75 appeared to perform better with false weak positives only in $1 / 7(14.3 \%)$ cases and $5 / 18$ (27.8\%) cases, respectively.

\section{Patterns of anti-PM-Scl100 and - 75 positivity}

There were 7 patients with dual positivity for anti-PMScl100 and anti-PM-Scl75, and all were true positives. Three cases had dual weak positive results and 4 had dual strong positive results. Four of these cases occurred in IIM patients with OM (scleromyositis) and 1 with amyopathic DM. The remaining 2 occurred in systemic sclerosis patients without myositis. Anti-PM-Scl100 occurred independently of anti-PM-Scl75 in 5 cases, 4 of which $(80.0 \%)$ were true positives. Anti-PM-Scl75, however, occurred independent of anti-PM-Scl100 in 19 cases where $12 / 19(63.2 \%)$ were true positives.

\section{Sensitivity, specificity, and factors associated with a positive result}

When considering individual Abs, specificity for the presence of a consistent diagnosis or disease subtype was generally high across all Abs (98.2-100.0\% for weak positives and $97.5-100.0 \%$ for strong positives; Supplementary Table 1). However, weak positive anti-SRP had the lowest specificity (97.0\%). A pre-test working diagnosis of IIM and a strong positive $\mathrm{Ab}$ result were significantly associated with true positivity (OR 50.8, 95\%CI 13.66-189.22, $p<0.001$ and OR 4.38, 95\%CI 2.32-8.26, $\mathrm{p}<0.001$, respectively) (Table 3 ). 
Table 3 Associations between true positive myositis-specific and myositis-associated autoantibodies and clinical factors

\begin{tabular}{|c|c|c|c|c|c|}
\hline Factor & Any true positive MSA/MAA & Any false positive MSA/MAA & OR & $\mathrm{P}$ & $\mathrm{Cl}$ \\
\hline Female gender & $78 \%(80 / 103)$ & $67 \%(50 / 75)$ & 1.74 & 0.10 & $0.89-3.39$ \\
\hline Mean age of onset (SD) & $50(13)$ & $52(15)$ & 0.99 & 0.35 & $0.96-1.01$ \\
\hline Pre-test working diagnosis of IIM & $59 \%(61 / 103)$ & $19 \%(14 / 75)$ & 50.8 & $<0.001$ & $13.66-189.22$ \\
\hline Biopsy in keeping with inflammatory myopathy & $50 \%(7 / 14)$ & $28 \%(7 / 25)$ & 2.57 & 0.18 & $0.66-10.06$ \\
\hline Myopathic EMG changes & $64 \%(21 / 33)$ & $43 \%(12 / 28)$ & 2.33 & 0.11 & $0.83-6.54$ \\
\hline Highest recorded CK (mean, SD) & $1377(3502)$ & $563(910)$ & 1.0 & 0.108 & $1.00-1.00$ \\
\hline Strong positive antibody result & $70 \%(72 / 103)$ & $\begin{array}{l}35 \% \\
26 / 75\end{array}$ & 4.38 & $<0.001$ & $2.32-8.26$ \\
\hline
\end{tabular}

MSA myositis-specific autoantibody, MAA myositis-associated autoantibody, OR odds ratio, $P$ p-value, $C l$ confidence intervals, $S D$ standard deviation, IIM idiopathic inflammatory myopathy, EMG electromyography, CK creatine kinase

\section{Discussion}

This study evaluated the clinical performance of the EUROIMMUN Inflammatory Myopathies 16 Ag LIA, a commonly used commercial assay. It is also the largest to report factors associated with true positive results. We showed that in expert-diagnosed IIM cases, $62.7 \%$ of patients had at least 1 identified Ab on LIA. A strong positive result and a high pre-test diagnosis of IIM was most associated with true positive results. This emphasises the value of an expert clinician's initial impression in reaching an IIM diagnosis and that LIA testing for IIM should be applied with caution in patients with low diagnostic suspicion for IIM.

A weak positive MSA was much more likely to be a false positive across all diagnostic groups (34/37 [91.9\%] false positives vs. $3 / 37$ [8.1\%] true positives). This was particularly true for weakly positive anti-SRP results which were all false positives in our study. Our study is in agreement with other recent publications which have also demonstrated that weak positives are more likely to be false [24]. Whilst weak positive MSAs were more likely to be false positives, specificity was high. Weak positive anti-SRP had the lowest specificity. Our results suggest that this LIA's accuracy may be improved if the threshold for defining weak positivity was increased, although this may vary according to each antibody on the assay [25].

We also found only 4/67 (5.9\%) IIM cases with multiple MSAs (excludes concurrent anti-Mi2A and anti-Mi2B, isoforms of Mi2 autoantibodies which coexist frequently [23]). Two of these cases only had 1 true MSA each and in the other 2 cases, all were false positive results. This is congruent with recent large cohort studies demonstrating mutual exclusivity of MSAs in IIM individuals [8] and highlights that when multiple MSAs are found in LIA testing, results should be treated with suspicion. Dual positivity for the MAAs anti-PM-Scl100 and anti-PM-Scl75, in contrast, improved the reliability of the results in both IIM and CTD without IIM cases.
Another notable finding was that a high proportion of IIM patients were seronegative (37.3\%). This number is comparable to recent findings from a large cohort of European IIM patients which found $38.3 \%$ of their cases to be seronegative [8]. A growing number of Abs currently not available in this LIA may be useful in the correct clinical context. For example, in addition to antiHMGCR, recent larger cohorts of IIM demonstrate that other emerging Abs such as anti-KS and anti-Zo are also useful in the diagnosis of IIM [8] .

Limitations of this study include data drawn from a single centre, although they represent a population of nearly 3 million people. Secondly, data were analysed retrospectively, and no specific additional review or tests were performed to confirm the diagnostic categorisation. Of the patients without CTD or IIM, most had at least 3 years of follow-up in their case notes, but it remains possible that positive $\mathrm{Ab}$ results may represent preclinical IIM or CTD. Additionally, negative Ab results were assumed to be true negatives. It is possible that some seronegative patients have detectable Abs via another method such as immunoprecipitation or have a hitherto undescribed Ab. Additionally, in cases where duplicate testing on the same patient occurred, we included only the most recent results. There is some evidence that certain MSAs might be lowered with treatment [26] so including only the most recent LIA result may have affected our results. However, clinicians seldom use the LIA for disease monitoring and the seven duplicates which occurred were more likely to be cases where the accuracy of first LIA test was in question. Finally, the final diagnoses made by the treating physicians could have been biased by the Ab results. However, most patients had several years of follow-up allowing for their diagnoses to be confirmed or reclassified over time and these final diagnoses were used in this study.

\section{Conclusions}

MSAs and MAAs are increasingly gaining importance in the diagnostic workup and management of IIMs. 
Clinicians may rely on commercially available LIAs to identify these Abs. This study describes the clinical utility of a widely used LIA and is the largest to report factors associated with true positive results. We demonstrated that strongly positive autoantibodies and a high pre-test likelihood of IIM are associated with true positive results on LIA. Specificity for individual MSAs on the LIA for IIM is high. Weak positive MSA results are more likely to be false than true positives. Our data suggests that cut-off values of the LIA may need to be redefined to increase its accuracy. Our work adds to the understanding of the increasing role of LIA testing for MSAs and MAAs in routine clinical practice and will support clinicians in understanding the relevance and implications of results.

\section{Supplementary information}

Supplementary information accompanies this paper at https://doi.org/10. 1186/s41927-020-00132-9.

Additional file 1: Table S1. Myositis specific and associated

autoantibodies specificities and categorisation by strength of positivity and true/false positive rate.

\section{Abbreviations}

Ab: Autoantibody; ASS: Anti-synthetase syndrome; CK: Creatine kinase; CTD: Connective tissue disease; DM: Dermatomyositis; EMG: Electromyography; IBM: Sporadic inclusion-body myositis; IIM: Idiopathic inflammatory myopathy; IMNM: Immune-mediated necrotising myopathy; LIA: Line blot immunoassay; MAA: Myositis associated autoantibody; MSA: Myositis specific autoantibody; OM: Overlap myositis; PM: Polymyositis; SRFT: Salford Royal NHS Foundation Trust

\section{Acknowledgements}

Not applicable.

\section{Authors' contributions}

FT collected, analysed, and interpreted the patient data and was a major contributor in writing the manuscript. JBL analysed and interpreted the patient data and was a major contributor in writing the manuscript. $\mathrm{HC}$ analysed and interpreted the patient data and contributed to the writing the manuscript. CR collected and analysed the patient data. SE interpreted the data. All authors read and approved the final manuscript.

\section{Funding}

This work was supported by a grant from the Medical Research Council (MR/ N003322/1). This report includes independent research supported by the NIHR Biomedical Research Centre Funding Scheme. The views expressed in this publication are those of the authors and not necessarily those of the NHS, the National Institute for Health Research or the Department of Health. The funding bodies played no role in the study design; collection, analysis, and interpretation of data; or composition of the manuscript.

\section{Availability of data and materials}

The datasets generated and/or analysed during the current study are available in the electronic health records employed by the Salford Royal NHS Foundation Trust.

\section{Ethics approval and consent to participate}

This study was performed as part of a quality improvement project evaluating LIA usage at SRFT. Case notes and other data were reviewed retrospectively without alteration to patient management. Given this context, and after consultation with the Health Research Authority (via www.hradecisiontools.org.uk) this study proceeded without further requirement for ethical authorization.
Consent for publication

Not applicable.

\section{Competing interests}

$\mathrm{HC}$ is an Associate Editor of BMC Rheumatology.

\section{Author details}

${ }^{1}$ Division of Rheumatology, University of British Columbia Division of Rheumatology, University of British Columbia, 802 - 1200 Burrard Street, Vancouver, BC V6Z 2C7, Canada. ${ }^{2}$ Complejo Hospitalario Universitario de A Coruña, Xubias de Arriba, 84, 15006 A Coruña, Spain. ${ }^{3}$ Department of Immunology, Salford Royal NHS Foundation Trust, 2nd floor, Turnberg Building, Stott Lane, Salford M68HD, UK. ${ }^{4}$ Centre for Musculoskeletal Research, School of Biological Sciences, Faculty of Biology, Medicine and Health, Manchester Academic Health Science Centre, The University of Manchester, Stopford Building, 99 Oxford Rd, Manchester M13 9PG, UK. ${ }^{5}$ Manchester Centre for Clinical Neurosciences, Salford Royal NHS Foundation Trust, Stott Lane, Salford M68HD, UK. ${ }^{6}$ National Institute for Health Research Manchester Biomedical Research Centre, Manchester University NHS

Foundation Trust, The University of Manchester, Manchester, UK.

${ }^{7}$ Department of Rheumatology, Salford Royal NHS Foundation Trust, Manchester Academic Health Science Centre, Stott Lane, Salford M68HD, UK. ${ }^{8}$ Centre for Musculoskeletal Research, Manchester Academic Health Science Centre, The University of Manchester, Manchester, UK.

Received: 14 January 2020 Accepted: 5 April 2020

Published online: 20 July 2020

\section{References}

1. Dalakas MC. Inflammatory muscle diseases. N Engl J Med. 2015;372(18): 1734-47.

2. Lundberg IE, Tjarnlund A, Bottai M, Werth VP, Pilkington C, Visser M, et al. 2017 European league against rheumatism/American College of Rheumatology classification criteria for adult and juvenile idiopathic inflammatory myopathies and their major subgroups. Ann Rheum Dis. 2017; 76(12):1955-64

3. Lundberg IE, Miller FW, Tjärnlund A, Bottai M. Diagnosis and classification of idiopathic inflammatory myopathies. J Intern Med. 2016;280(1):39-51.

4. Marguerie C, Bunn CC, Beynon HL, Bernstein RM, Hughes JM, So AK, et al. Polymyositis, pulmonary fibrosis and autoantibodies to aminoacyl-tRNA synthetase enzymes. Q J Med. 1990;77(282):1019-38.

5. Pinal-Fernandez I, Casal-Dominguez M, Mammen AL. Immune-mediated necrotizing myopathy. Curr Rheumatol Rep. 2018;20(4):21.

6. Mammen AL, Casciola-Rosen L, Christopher-Stine L, Lloyd TE, Wagner KR. Myositis-specific autoantibodies are specific for myositis compared to genetic muscle disease. Neurology(R) neuroimmunology \& neuroinflammation. 2015:2(6):e172.

7. Betteridge Z, McHugh N. Myositis-specific autoantibodies: an important tool to support diagnosis of myositis. J Intern Med. 2016;280(1):8-23.

8. Betteridge Z, Tansley S, Shaddick G, Chinoy H, Cooper RG, New RP, et al. Frequency, mutual exclusivity and clinical associations of myositis autoantibodies in a combined European cohort of idiopathic inflammatory myopathy patients. J Autoimmun. 2019;101:48-55.

9. Mariampillai K, Granger B, Amelin D, Guiguet M, Hachulla E, Maurier F, et al. Development of a New classification system for idiopathic inflammatory myopathies based on clinical manifestations and myositis-specific autoantibodies. JAMA Neurol. 2018;75(12):1528-37.

10. Oldroyd A, Sergeant JC, New P, MCHugh NJ, Betteridge Z, Lamb JA, et al. The temporal relationship between cancer and adult onset antitranscriptional intermediary factor 1 antibody-positive dermatomyositis. Rheumatology. 2018.

11. Allenbach $Y$, Benveniste $\mathrm{O}$, Goebel HH, Stenzel W. Integrated classification of inflammatory myopathies. Neuropathol Appl Neurobiol. 2017;43(1):62-81.

12. Anquetil $C$, Boyer $O$, Wesner N, Benveniste O, Allenbach $Y$. Review: myositisspecific autoantibodies, a cornerstone in immune-mediated necrotizing myopathy. Autoimmun Rev. 2019;10:10.

13. Cruellas MG, Viana VS, Levy-Neto M, Souza FH, Shinjo SK. Myositis-specific and myositis-associated autoantibody profiles and their clinical associations in a large series of patients with polymyositis and dermatomyositis. Clinics. 2013;68(7):909-14 
14. Ghirardello A, Zampieri S, Tarricone E, laccarino L, Bendo R, Briani C, et al. Clinical implications of autoantibody screening in patients with autoimmune myositis. Autoimmunity. 2006;39(3):217-21.

15. Gofrit SG, Yonath H, Lidar M, Shoenfeld Y, Kivity S. The clinical phenotype of patients positive for antibodies to myositis and myositis-related disorders. Clin Rheumatol. 2018;37(5):1257-63.

16. Li L, Wang H, Wang Q, Wu C, Liu C, Zhang Y, et al. Myositis-specific autoantibodies in dermatomyositis/polymyositis with interstitial lung disease. J Neurol Sci. 2018:397:123-8.

17. Pinal-Fernandez I, Casal-Dominguez M, Huapaya JA, Albayda J, Paik JJ, Johnson C, et al. A longitudinal cohort study of the anti-synthetase syndrome: increased severity of interstitial lung disease in black patients and patients with anti-PL7 and anti-PL12 autoantibodies. Rheumatology. 2017;56(6):999-1007.

18. Gunawardena H, Betteridge ZE, McHugh NJ. Myositis-specific autoantibodies: their clinical and pathogenic significance in disease expression. Rheumatology. 2009:48(6):607-12.

19. Ghirardello A, Bettio S, Bassi N, Gatto M, Beggio M, Lundberg I, et al. Autoantibody testing in patients with myositis: clinical accuracy of a multiparametric line immunoassay. Clin Exp Rheumatol. 2017;35(1):176-7.

20. Ghirardello A, Rampudda M, Ekholm L, Bassi N, Tarricone E, Zampieri S, et al. Diagnostic performance and validation of autoantibody testing in myositis by a commercial line blot assay. Rheumatology. 2010;49(12):2370-4.

21. Parker MJS, Oldroyd A, Roberts ME, Lilleker JB, Betteridge ZE, McHugh NJ, et al. The performance of the European league against rheumatism/ American College of Rheumatology idiopathic inflammatory myopathies classification criteria in an expert-defined 10 year incident cohort. Rheumatology. 2018.

22. Fujimoto M. Dermatomyositis: myositis-specific autoantibodies and skin manifestations. Clinical and Experimental Neuroimmunology. 2012;3(2):74-84.

23. Richards M, Garcia-De La Torre I, Gonzalez-Bello YC, Vazquez-Del Mercado M, Andrade-Ortega L, Medrano-Ramirez G, et al. Autoantibodies to Mi-2 alpha and $\mathrm{Mi}-2$ beta in patients with idiopathic inflammatory myopathy. Rheumatology. 2019;58(9):1655-61.

24. Tansley SL, Li D, Betteridge ZE, McHugh NJ. The reliability of immunoassays to detect autoantibodies in patients with myositis is dependent on autoantibody specificity. Rheumatology. 2020.

25. Mecoli CA, Albayda J, Tiniakou E, Paik JJ, Zahid U, Danoff SK, et al. Myositis Autoantibodies: A Comparison of Results From the Oklahoma Medical Research Foundation Myositis Panel to the Euroimmun Research Line Blot. Arthritis \& rheumatology (Hoboken, NJ). 2020;72(1):192-4.

26. Aggarwal R, Oddis CV, Goudeau D, Koontz D, Qi Z, Reed AM, et al. Autoantibody levels in myositis patients correlate with clinical response during B cell depletion with rituximab. Rheumatology. 2016;55(6):991-9.

\section{Publisher's Note}

Springer Nature remains neutral with regard to jurisdictional claims in published maps and institutional affiliations.

Ready to submit your research? Choose BMC and benefit from:

- fast, convenient online submission

- thorough peer review by experienced researchers in your field

- rapid publication on acceptance

- support for research data, including large and complex data types

- gold Open Access which fosters wider collaboration and increased citations

- maximum visibility for your research: over $100 \mathrm{M}$ website views per year

At $\mathrm{BMC}$, research is always in progress.

Learn more biomedcentral.com/submissions 